\title{
Spatial-temporal characteristics of perceptual organization following acquired brain injury
}

\author{
DANIEL D. KURYLO ${ }^{1}$, RICHARD WAXMAN ${ }^{2,3}$, \& OZLEM KEZIN ${ }^{1}$ \\ ${ }^{1}$ Psychology Department, Brooklyn College CUNY, Brooklyn, NY, USA, ${ }^{2}$ Psychology, Touro College, Brooklyn, NY, \\ USA, and ${ }^{3}$ Peninsula Hospital Center, Queens, NY, USA
}

(Received 30 August 2004; accepted 25 August 2005)

\begin{abstract}
Objective: To assess characteristics of perceptual organization following brain injury.

Research design: A repeated measures between groups design.

Methods: Perceptual thresholds were compared between 10 persons with brain injury and 10 demographically matched healthy control subjects. Ten young control subjects were also tested to evaluate effects of normal ageing on these processes. Participants viewed stimuli on a computer monitor in which regularities existed in either the spatial or temporal domain. Psychophysical measurements were made of perceptual thresholds, as well as time required to process stimuli.

Results: Relative to age-matched control subjects, patients were impaired on all measures, with greatest deficits in conditions that contained a temporal component. Impairment did not occur with normal ageing.

Conclusions: These results reflect cognitive impairment resulting from reduced cortical integrative function as well as reduced information processing speed. Such deficits at this level of processing likely impact subsequent perceptual function, such as object recognition.
\end{abstract}

Keywords: Perceptual organization, grouping, gestalt, proximity, TBI, CVA

\section{Introduction}

Perceptual organization is a basic component of visual processing in which individual elements of a visual scene are resolved into a series of unified forms. Perceptual organization is an essential process that serves to organize stimuli in preparation for high-order visual functions. Disruption at the level of perceptual organization will thereby interfere with the subsequent processing of object recognition [1] or exacerbate deficits in other cognitive processes, such as memory [2]. Because of its relationship to vision and other cognitive abilities, it is important to understand the consequence of cerebral injury on perceptual organizational capacities.

Perceptual organization is based upon regularities that exist among stimulus features and may be established from a variety of spatial and temporal relationships. Spatial relationships include proximity and regularity in position, as well as similarity in luminance, shape or colour. Temporal relationships include coherence in motion or luminance modulation. With complex stimuli in natural scenes, perceptual organization is based upon the interaction of multiple stimulus features, which provide sources for multiple perceptual strategies.

Disturbances to high-order visual function are associated with extra-striate areas, particularly in cases of right hemispheric damage. Impaired object recognition, which results from injury to inferotemporal areas [3, 4], has been associated with reduced ability to establish relationships among stimulus features [5] or with a reduced field of attention in which stimulus features may be integrated [6]. Although feature integration is a basic component of high-order visual function, perceptual organization has not been examined following brain injury.

Correspondence: Daniel D. Kurylo, PhD, Psychology Department, Brooklyn College CUNY, 2900 Bedford Avenue, Brooklyn, NY 11210, USA. Tel: 718-951-5969. Fax: 718-951-4814. E-mail: dkurylo@brooklyn.cuny.edu 
The focus of this study is to determine characteristics of perceptual organization following brain injury. To accomplish this, performance by a group of persons with brain injury was compared to agematched control subjects. Because some components of perceptual organization are compromised with age [7-9], brain injury may exacerbate functional decline found in normal ageing. Therefore, a group of young control subjects was also tested to track the effects of normal ageing on perceptual measurements made here.

Perceptual organization requires the integration of information across the cortical surface. Damage to fibres connecting processing modules will thereby disrupt cortical integration. Based upon this, it is hypothesized that deficits will be found in persons with brain injury for integration across spatial position. In addition, generalized slowing of processing following brain injury $[10,11]$ should interfere with integrating information over time, such as with stimuli presented in rapid sequence. It is, therefore, further hypothesized that perceptual organization associated with temporal integration will also be impaired in the patient group. Such deficits should be apparent in threshold levels at which integration takes place (Grouping Threshold), as well as in the time required to complete processing (Masking Threshold).

\section{Methods}

\section{Subjects}

Thirty participants from three subject groups participated in the study. Participants from the acquired brain injury group were drawn from the Outpatient Ambulatory Care Unit of Peninsula Hospital Center, Queens, NY. Patients had received a basic ophthalmologic examination as part of their outpatient evaluation and were confirmed to be free from significant ophthalmologic disorders, including visual field restrictions. The patient group included right-hemisphere cerebral vascular accident (CVA) ( $n=5$; mean age $=65.8$ years $)$, left-hemisphere CVA $(n=2$; mean age $=54.0$ years $)$, and traumatic brain injury (TBI) $(n=3$; mean age $=52.0$ years). The mean duration since the time of injury was 7.2 years. Elderly control subjects (EC) $(n=10$; mean age $=58.4$ years) consisted of volunteers with no history of neurological disorder. Patients and EC subjects did not differ significantly in age $(t(18)=0.27 ; p=0.79)$. Young control subjects (YC) $(n=10)$ consisted of college students who participated in the study as part of a course requirement. This study was conducted in accordance with the Institutional Review Board for Human Research of Brooklyn College as well as from Peninsula Hospital Center.

\section{General procedure}

Participants viewed stimuli that could be perceptually grouped as either vertical or horizontal lines. Participants received four tests of perceptual organization that were based upon either spatial proximity or temporal contrast (flicker). For each stimulus condition, psychophysical measurements were made of grouping thresholds, which represent the limits of perceptual organization, as well as stimulus masking thresholds, which represent time necessary to complete the process. For grouping threshold measurements, the relative proximity or the level of similarity was progressively reduced until stimuli could no longer be perceptually organized. For stimulus masking measurements, stimuli were followed by a pattern mask and the stimulus duration was progressively reduced until the grouping pattern became ambiguous, thereby determining processing time required to establish perceptual organization.

To minimize confounding factors associated with slowed decision-making or motor response, reaction time was not a factor and participants were instructed to optimize accuracy. In addition, responses were based upon a forced-choice procedure in order to preclude possible response bias that may distinguish subject groups.

\section{Stimuli}

Stimuli were briefly presented on a computer monitor. Stimuli were composed of a grid of elements that subtended a $19.3^{\circ}$ square field. Stimulus duration was linked to the display's vertical synchronization signal. Stimulus elements were organized either vertically or horizontally, selected randomly on each trial, which elicited the perception of a series of either vertical or horizontal lines.

Proximity. For the proximity condition, stimulus elements were solid squares, $0.35^{\circ}$ on a side. Elements were aligned and spaced at regular intervals, differing in separation between the vertical and horizontal orientation (Figure 1(a)). Element separation along the less proximal orientation was fixed at $3.03^{\circ}$, whereas separation along the more proximal orientation ranged from $2.02-3.03^{\circ}$. Metrics of the proximity cue are described in terms of relative separation (Figure 1(c)). For example, a relative separation of $110 \%$ indicates that elements along the fixed orientation were $110 \%$ of the separation of the more proximal orientation. At the beginning of each measurement series, the relative separation 
(a)

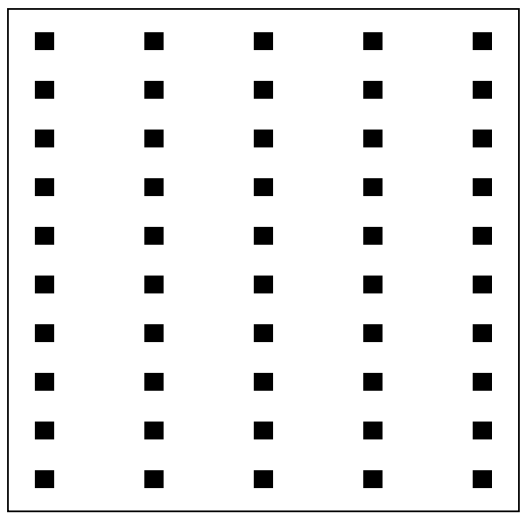

(c)

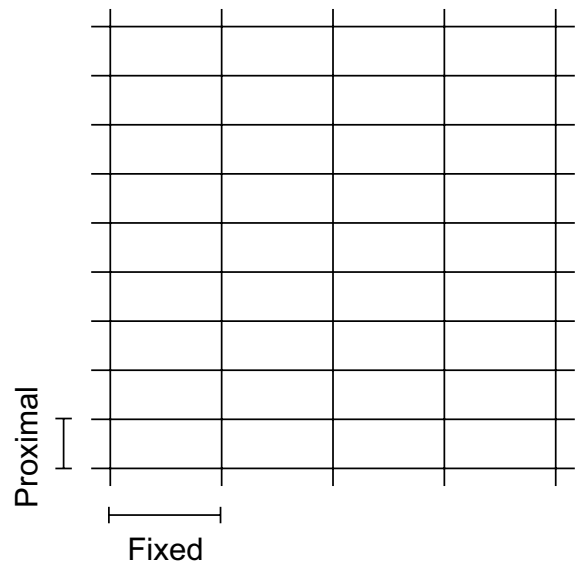

(b)

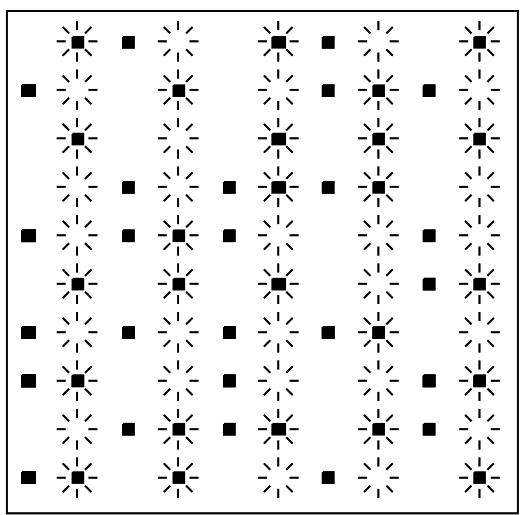

(d)

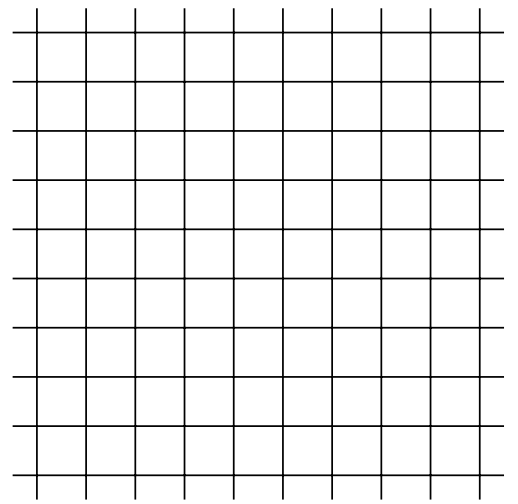

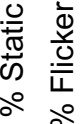

Figure 1. Stimuli and associated metrics. For each trial, grouping cues were randomly assigned to either the horizontal or vertical orientation. For examples shown here, grouping cues are assigned to the vertical orientation. (a) Proximity condition: Elements along the horizontal orientation are more separated than those along the vertical. (c) Metrics are defined in terms of the relative element separation (fixed separation/proximal separation). (b) Flicker condition: Flickering elements are depicted by radiating line; other elements are static throughout presentation. Shown here is a single frame in which every other column contains flickering elements, alternating with columns of static elements. (d) Metrics for the flicker condition are described in terms of percentage of elements that flicker along the flickering columns, which is equal to the percentage of static elements along alternate columns. Shown here is the $100 \%$ similarity level.

was $150 \%$. Across trials, relative separation was progressively reduced, in increments of $5 \%$, until proximity no longer served as a cue for grouping.

Flicker. For the flicker condition, stimulus elements were solid squares, $0.35^{\circ}$ on a side, that either flickered or remained on. Square wave modulation of flickering elements cycled three times at $7.5 \mathrm{~Hz}$. For the vertical condition, every other column contained flickering elements and alternate columns contained static elements (Figure 1(b)). For the horizontal condition, elements were organized similarly, but along rows. Elements along the flickering columns (or rows) were counterbalanced in phase and half of the elements along static columns were visible. The phase of flickering elements and the distribution of static elements were selected randomly on each trial. Therefore, on each frame, half of the possible elements were present, distributed randomly across the array. These constraints precluded the possibility that element proximity or density on a single frame could be used for perceptual organization. At the beginning of each measurement series, static and flickering elements were sorted between alternating columns (or rows). As trials progressed, the level of organization, described in terms of percentage similarity (Figure 1(d)), progressively decreased in increments of $2 \%$. 


\section{Grouping thresholds}

For grouping thresholds, measurements were made of the lowest relative separation (proximity condition) or lowest percentage of similarity (flicker condition) necessary to perceptually organize the stimulus. Below this level, organization became ambiguous and stimuli did not produce a reliable grouping pattern. Thresholds were determined by means of a two-alternative forced-choice staircase procedure. Stimulus difficulty level was increased after two consecutive correct responses and decreased after a single incorrect response, thereby converging on a level at which subjects respond correctly with a long-run probability of $71 \%$ [12]. Thresholds were based upon the mean of eight reversals from two descending series.

Procedure. Participants fixated a central target on the computer monitor at a viewing distance of $46 \mathrm{~cm}$. Following a delay of $500 \mathrm{~ms}$, stimuli appeared for $500 \mathrm{~ms}$. On each trial, the vertical or horizontal condition was randomly assigned. Following stimulus presentation, subjects indicated whether the stimulus was organized as a series of vertical or horizontal lines. Responses were made either verbally, signalling with hand motions or pointing to picture representations of the choices. Responses were then entered into the computer by the experimenter. Reaction time was not a factor and subjects were instructed to maximize the accuracy and not the speed of their response. For each test, subjects first received a demonstration and then a series of practice trials in order to become familiar with the stimulus and procedure. Following the demonstration and practice, threshold measurements were made. Stimulus generation, data collection and contingency algorithms were controlled by computer.

\section{Masking thresholds}

A backward pattern mask was used to determine the duration of processing necessary to establish perceptual organization [13]. The test stimulus was followed by a pattern mask, which served to disrupt processing of the test stimulus. Test stimuli were immediately followed by the pattern mask, thereby maintaining experimental control over the test stimulus without introducing post-stimulus effects [14]. Stimulus duration was progressively reduced across trials until the organization of the stimulus could no longer be determined. Under these conditions, other stimulus characteristics, such as element shape, were identifiable, but perceptual organization failed to be achieved. Masks were, therefore, not intended to eliminate detection of stimulus features, but to interfere with processes associated with perceptual organization.

Test stimulus. Test stimuli for the proximity and flicker conditions were the same as those previously described. In both cases, relative separation was fixed at $150 \%$ (proximity condition) or $100 \%$ similarity (flicker condition).

Masking stimulus. The masking stimulus was an $8 \times 8$ array of crosses (plus signs). The array of crosses contained robust horizontal and vertical co-linearity cues and effectively disrupted perceptual organization of test stimuli. Stimulus sequence for the flicker masking condition is shown in Figure 2.

Procedure. Participants fixated a central point, which was followed after $500 \mathrm{~ms}$ by the test stimulus. Immediately following the offset of the test stimulus, the mask appeared for $200 \mathrm{~ms}$. After viewing both stimuli, participants indicated whether the test stimulus appeared to be organized as a series of vertical or horizontal lines. At the beginning of each trial series, the test stimulus duration was $600 \mathrm{~ms}$. Across trial, stimulus duration was progressively reduced in increments of $14.29 \mathrm{~ms}(70 \mathrm{~Hz}$ temporal resolution). Thresholds were again based upon the mean of eight reversals from two descending series.

\section{Control conditions}

Each psychophysical measurement was accompanied by a control condition which contained solid vertical or horizontal lines. These trials were introduced in order to monitor subjects' ability to discriminate figures constructed of solid lines. Performance on the control conditions provide an assessment of subjects' ability to understand the requirements of the tasks, to perceive and discriminate each pair of stimuli and to respond appropriately. Impaired performance on experimental conditions is, therefore, attributable to deficits specific to perceptual organization and not other cognitive factors.

\section{Results}

\section{Proximity condition}

Analysis of variance (ANOVA) indicated subject groups differed significantly for proximity grouping thresholds $(F(2,27)=7.31 ; p<0.01)$ (Figure $3(a))$. Post hoc pair-wise comparisons indicated that the patient group $(M=1.40 \%)$ required significantly greater relative separation $(p<0.01)$ to perform grouping compared to the EC group $(M=1.11 \%)$. 


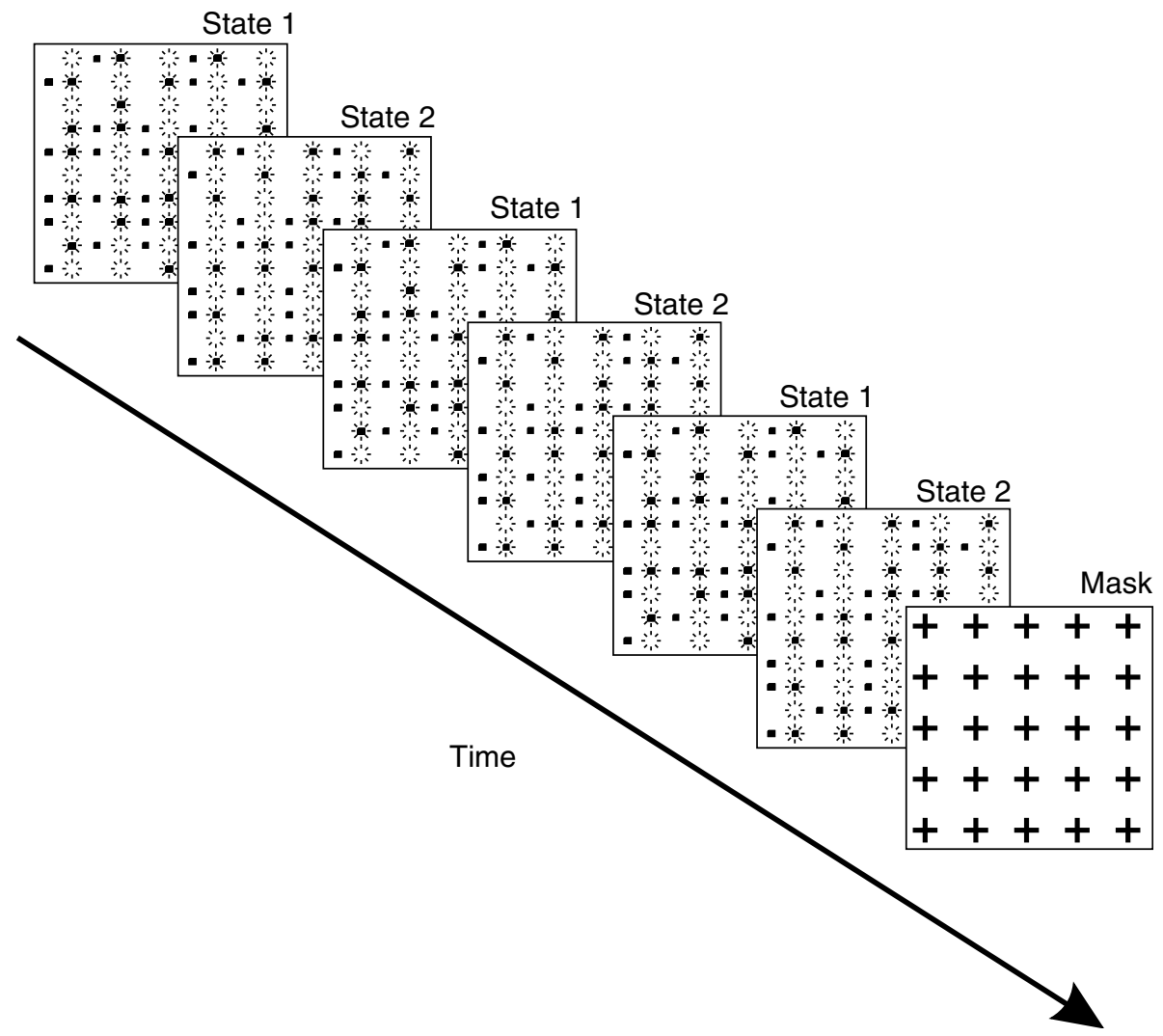

Figure 2. Temporal sequence of the flicker condition with the mask for a single stimulus presentation. Six frames appear sequentially in which flickering elements alternate between states, which is then immediately followed by the pattern mask.

(a)

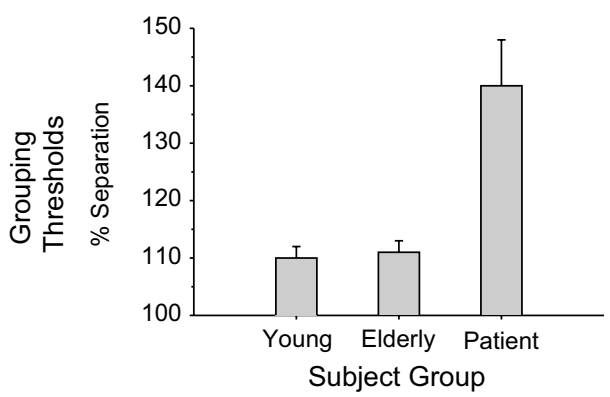

(c)

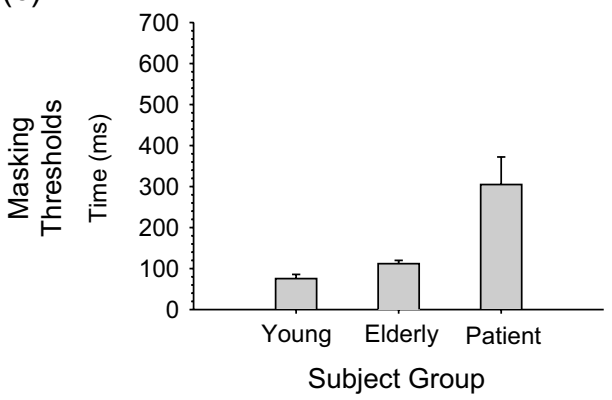

(b) $\quad$ Flicker

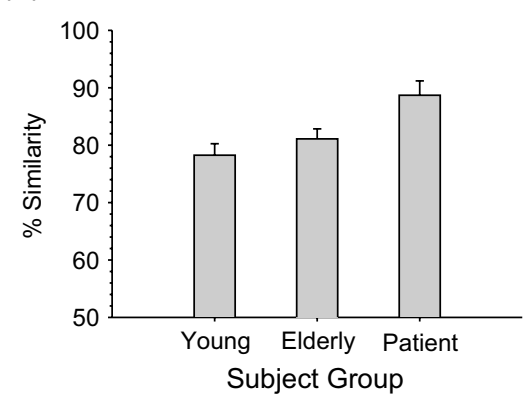

(d)

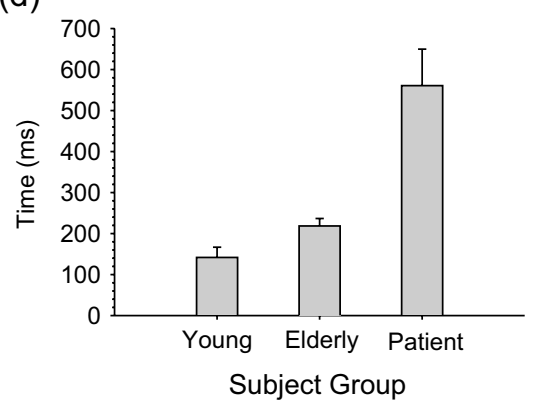

Figure 3. Grouping and masking thresholds (mean and SEM) for each subject group. Grouping thresholds (a) and masking thresholds (c) for the proximity condition; Grouping thresholds (b) and masking thresholds (d) for the flicker condition. 
Young and elderly subjects did not differ significantly on proximity grouping thresholds. For proximity masking threshold, subject groups differed significantly $(F(2,27)=12.04 ; \quad p<0.01)$ (Figure 3(c)), in which the patient group $(M=325.19 \mathrm{~ms})$ required greater time to complete perceptual organization than elderly controls $(M=74.184 \mathrm{~ms}) \quad(p<0.05)$. Young and elderly control subjects did not differ significantly for proximity masking thresholds.

Flicker condition. For organization based upon flicker, subject groups differed significantly on grouping threshold $(F(2,27)=19.96 ; p<0.01)$ (Figure 3(b)). The patient group $(M=91.24 \%)$ required significantly greater stimulus organization to establish grouping $(p<0.01)$ relative to elderly controls $(M=81.04 \%)$. Flicker grouping thresholds did not differ significantly between age groups (YC group, $M=78.51 \%$ ). For masking thresholds, subject groups differed significantly $(F(2,26)=$ $17.719 ; p<0.01$ ) (Figure 3(d)), in which the patient group $(M=644.45 \mathrm{~ms})$ required greater durations to perform grouping $(p<0.01)$ than the EC group $(M=169.53 \mathrm{~ms})$. Flicker masking thresholds did not differ significantly between age groups (YC group, $M=109.67 \mathrm{~ms}$ ). Examining individual performance on the flicker task, four of 13 patients displayed grouping thresholds of greater than $94 \%$ similarity, which represents a level expected from chance performance. Subjects performing at this level were, therefore, unable to establish grouping with even the most salient stimulus. In order to examine patient group performance without the impact of these subjects, an ANOVA was repeated with the remaining subjects. This sub-group analysis also indicated significantly elevated grouping $(F(2,22)=3.25 ; p<0.05)$ and masking thresholds $(F(2,22)=10.513 ; p<0.01)$ for the patient group compared to elderly controls.

\section{Control condition}

For all participants, performance on control trials remained at $100 \%$ correct throughout the testing session. Therefore, no participant was excluded from data analysis.

\section{Sub-group comparisons}

For both the Proximity and Flicker conditions, patient sub-groups (R-, L- CVA and TBI) performed qualitatively similar to each other and in each case differed from control subjects.

\section{Discussion}

These results identify impairment at the level of perceptual organization that results from injury outside striate cortex. Deficits are evident as elevated grouping thresholds, as well as extended processing time. In this regard, deficits are found with perceptual integrative function, as well as reduction in information processing speed. Accurate performance on the control (solid line) trials, as well as the highest proximity level for the proximity threshold condition, verifies that patients understood task requirements and were capable of visual discrimination. Furthermore, accuracy on these conditions suggest that elevated thresholds do not represent general decline in perceptual or other cognitive abilities, but instead reflect impairment to perceptual integrative function and processing speed. In addition, performance on these tasks did not decline with normal ageing. In this regard, impairment found here does not appear to reflect an exacerbation of normal decline with ageing, but is instead attributed to disruption specific to injury.

The most significant impairments occurred for those tasks that contained a temporal component. Speed of information processing is often evaluated by reaction-time tasks, which include cognitive processing such as discrimination and decisionmaking, as well as preparation and execution of motoric components. The backward pattern mask procedure used here isolates analysis of the stimulus, thereby specifying stimulus processing duration. In addition, slowing of information processing following head injury [15] has been associated with impaired executive functioning [16] or with slowed activation of memory [11]. The procedure used here did not introduce unexpected stimuli and the repetitiveness and predictability of the task minimized demands on executive function and memory. Processing speed reduction found here is, therefore, specific to stimulus processing and may be accounted for by increased demands placed on neurons that serve to integrate information. With a reduced number of units operating within a system, local circuits may require extended time to complete functions. Similarly, reduced temporal integration may account for deficits in the flicker condition. Insufficient time to process individual frames would interfere with identifying associations across frames, thereby disrupting temporal integration.

Perceptual organization is often addressed in terms of hierarchical visual analysis, in which processing of local features is distinguished from processing of the global configuration of stimuli. This distinction has been associated with hemispheric differentiation, such that left-hemisphere damage produces greater impairment on analysis 
of local features, whereas right-hemisphere damage produces greater impairment on global analysis $[17,18]$. The integrity of global analysis following brain injury is evaluated by several neuropsychological measures. Disruption of perceptual organization skills resulting from brain injury have been associated with reduced performance on the Rey Complex Figure Test [19]. Similarly, performance on the Hooper Visual Organization Test, used to assess visuospatial abilities, is reduced with brain injury, particularly with right hemisphere damage $[20,21]$. Deficits in the processing of global features are also reflected by errors in overall configuration of the Block Design sub-test of the WAIS-R, which has also been associated with right hemisphere lesions $[22,23]$. A specific index of Perceptual Organization is now provided by three sub-tests of the WAIS-III (Picture Completion, Block Design and Matrix Reasoning). Psychophysical thresholds obtained here provide details of constituent characteristics of perceptual organization deficits. Results indicate an impairment in integrating stimulus elements that are associated in spatial position or that are modified across time.

In this regard, impaired perceptual organization following brain injury may reflect a general reduction in cortical integrative function. Cortical integration is mediated by connections that co-ordinate processing modules. Such connections include lateral processing among cortical columns [24, 25] and reciprocal cortico-thalamic routes [26]. Long-range cross-cortical connections also allow integration among hierarchical areas [27], including feedback mechanisms [28]. Disruption of cortical processing following brain injury is, therefore, likely to impact connections for cortical integration. Under these circumstances, many aspects of visual processing remain intact, whereas integrative processes will be impaired. Because perceptual organization involves the integration and co-ordination of information, it is likely to be particularly vulnerable to disruption of cortical integration.

The clinical significance of these findings relates to the interpretation of neuropsychological measurements that contain an integrative component. Because perceptual organization occurs at an early or intermediate level of visual processing, impairment is likely to degrade performance on other perceptual and cognitive functions that occur at a later stage of processing. High-order visual processing, including spatial relationships and the identification of common objects and familiar faces may either be impaired or delayed by a decline in perceptual organization. Similarly, perceptual organization can impact performance on visual shortterm memory [2], visual search [8] and selective attention [29]. With the clinical evaluation of these and other cognitive functions, consideration should be made of the possible contribution of impaired perceptual organization. In addition, cognitive functions that include temporal components, such as evaluating information that alters over time, may also be degraded by disrupted perceptual organization.

Impairments identified here apply to experimentally controlled stimuli that allow evaluation of isolated perceptual components. For natural viewing conditions, which contain a complex interaction of multiple stimulus features, patients may benefit from stimulus features less affected at the level of perceptual organization. For example, exaggerated spatial proximity and extended display times that minimize reliance on temporal integration may improve object identification, thereby improving the level of functioning and the care of patients.

\section{Acknowledgement}

This work was supported (in part) by the City University of New York PSC-CUNY Research Award Program.

\section{References}

1. Uttal WR. On seeing forms. Hillsdale, N.J.: Lawrence Erlbaum; 1988 .

2. Humphrey DG, Kramer AF. Age-related differences in perceptual organization and selective attention: implications for display segmentation and recall performance. Experimental Aging Research 1999;25:1-26.

3. Lansdell H. Effect of extent of temporal lobe ablations on two lateralized deficits. Physiology and Behavior 1968;3:271-273.

4. Newcombe F, Russell WR. Dissociated visual perceptual and spatial deficits in focal lesions of the right hemisphere. Journal of Neurology, Neurosurgery, and Psychiatry 1969;32:73-81.

5. Layman S, Greene E. The effects of stroke on object recognition. Brain and Cognition 1988;7:87-114.

6. Stark ME, Grafman J, Fertig E. A restricted 'spotlight' of attention in visual object recognition. Neuropsychologia 1997;35:1233-1249.

7. Kurylo DD, Allan WC, Collins TE, Barron J. Perceptual organization based upon spatial relationships in Alzheimer's disease. Journal of Behavioural Neurology 2003;14:19-28.

8. Gilmore GC, Tobias TR, Royer FL. Aging and similarity grouping in visual search. Journal of Gerontology 1985;40:586-592.

9. Farkas MS, Hoyer WJ. Processing consequences of perceptual grouping in selective attention. Journal of Gerontology 1980;35:207-216.

10. Zahn TP, Mirsky AF. Reaction time indicators of attention deficits in closed head injury. Journal of Clinical and Experimental Neuropsychology 1999;21:352-367.

11. Tromp E, Mulder T. Slowness of information processing after traumatic head injury. Journal of Clinical and Experimental Neuropsychology 1991;13:821-830. 
12. Levitt H. Transformed up-down methods in psychoacoustics. Journal of the Acoustical Society of America 1971;49:467-477.

13. Kurylo DD, Time course of perceptual grouping, Perception and Psychophysics 1997;59:142-147.

14. Felsten G, Wasserman GS. Visual masking: mechanisms and theories. Psychological Review 1980;88:329-354.

15. Fisher DC, Ledbetter MF, Cohen NJ, Marmor D, Tulsky DS. WAIS-III and WMS-III profiles of mildly to severely brain-injured patients. Applied Neuropsychology 2000;7:126-132.

16. Madigan NK, Deluca J, Diamond BJ, Tramontano G, Averill A. Speed of information processing in traumatic brain injury: modality-specific factors. The Journal of Head Trauma Rehabilitation 2000;15:943-956.

17. Delis DC, Kiefner MG, Fridlund AJ. Visuospatial dysfunction following unilateral brain damage: dissociations in hierarchical and hemispatial analysis. Journal of Clinical and Experimental Neuropsychology 1991;10:421-431.

18. Lamb MR, Robertson LC, Knight RT. Component mechanisms underlying the processing of hierarchically organized patterns: interferences from patients with unilateral cortical lesions. Journal of Experimental Psychology: Learning, Memory and Cognition 1990;16:471-483.

19. Ashton VL, Donders J, Hoffman NM. Rey Complex Figure Test performance after traumatic head injury. Journal of Clinical and Experimental Neuropsychology 2005;27:55-64.

20. Fitz AG, Conrad PM, Horn DL, Sarff PL, Majovski LV. Hooper Visual Organization Test performance in lateralized brain injury. Archives of Clinical Neuropsychology 1992;7:243-250.
21. Nadler JD, Grace J, White DA, Butters MA, Malloy PF. Laterality differences in quantitative and qualitative Hooper Performance. Archives of Clinical Neuropsychology 1996;11:223-229.

22. Kramer JH, Kaplan E, Blusewicz MJ, Preston KA. Visual hierarchical analysis of Block Design configural errors. Journal of Clinical and Experimental Neuropsychology 1991;13:455-465.

23. Wilds MC, Boake C, Sherer M. Wechsler Adult Intelligence Scale-Revised Block Design broken configuration errors in nonpenetrating traumatic brain injury. Applied Neuropsychology 2000;7:208-214.

24. Bringuier V, Chavane F, Glaeser L, Fregnac Y. Horizontal propagation of visual activity in the synaptic integration field of area 17 neurons. Science 1990;283: 695-699.

25. Gilbert CD, Wiesel TN. Intrinsic connectivity and receptive field properties in visual cortex. Vision Research 1985;25:365-374.

26. Rauschecker JP, von Grünau MW, Poulin C. Thalamocortical connections and their correlation with receptive field properties in the cat's lateral suprasylvian visual cortex. Experimental Brain Research 1987;67:100-112.

27. Polat U. Functional architecture of long-range perceptual interactions. Spatial Vision 1999;12:143-162.

28. Lamme VA, Supèr H, Spekreijse H. Feedforward, horizontal, and feedback processing in the visual cortex. Current Opinion in Neurobiology 1998;8: 529-535.

29. Maylor EA, Lavie N. The influence of perceptual load on age differences in selective attention. Psychology and Aging 1998;13:563-573. 